\title{
Room-temperature dephasing in InAs/GaAs quantum dots
}

Borri, Paola; Langbein, Wolfgang; Hvam, Jørn Märcher; Mao, M. -H.; Heinrichsdorff, F.; Bimberg, Dieter

\section{Published in:}

Proceedings of QELS'99

Link to article, DOI:

10.1109/QELS.1999.807152

Publication date:

1999

Document Version

Publisher's PDF, also known as Version of record

Link back to DTU Orbit

Citation (APA):

Borri, P., Langbein, W., Hvam, J. M., Mao, M. -H., Heinrichsdorff, F., \& Bimberg, D. (1999). Room-temperature dephasing in InAs/GaAs quantum dots. In Proceedings of QELS'99 (pp. QTuC2). IEEE.

https://doi.org/10.1109/QELS.1999.807152

\section{General rights}

Copyright and moral rights for the publications made accessible in the public portal are retained by the authors and/or other copyright owners and it is a condition of accessing publications that users recognise and abide by the legal requirements associated with these rights.

- Users may download and print one copy of any publication from the public portal for the purpose of private study or research.

- You may not further distribute the material or use it for any profit-making activity or commercial gain

- You may freely distribute the URL identifying the publication in the public portal 


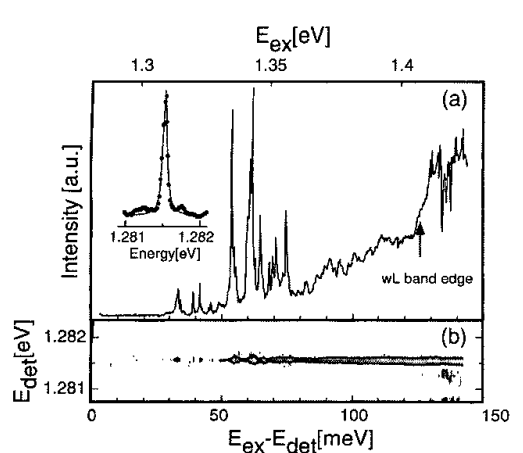

QTuC1 Fig. 1. (a) Typical PLE spectrum of a PL feature as shown in the inset. The PL was excited by a Ti:sapphire laser with a frequency resolution of $\sim 10 \mathrm{GHz}$ and an excitation density of $\sim 10 \mathrm{~W} / \mathrm{cm}^{2}$. The horizontal bottom axis represents the relaxation energy $\left(E_{\text {rel }}\right)$, which corresponds to the difference between excitation and detection energies $\left(E_{e x c}-E_{d e t}\right)$. Solid line in the inset indicates a Lorenzian fit to the PL data with $\mathrm{E}_{\mathrm{ex}}=1.43 \mathrm{eV}$. The luminescence linewidth is estimated to be $\sim 70 \mu \mathrm{eV}$. (b) PL intensities corresponding to the data in (a) are plotted as a function of detection and excitation energy.

When the relaxation energy matches an available phonon energy, the emitted photons fulfil the resonance condition by the emission of a LO phonon. Therefore, the observed PLE resonances are attributed to resonant Raman features.

These results provide new insights into the relaxation process in SAQDs as follows: the carriers can relax within continuum states, and make transitions to the excitonic ground state by resonant phonon scattering before PL emission, thus allowing for the intense PL peak observed for SAQDs.

The authors thank S. Mononobe and M. Ohtsu for their kind arrangement on the fiber probe. This work is supported in part by the Research for the Future Program of the Japan Society for the Promotion of Science (Project No. JSPS-RFTF96P00201), Grant in-aid of Priority Area by Ministry of Education, Science and Culture, and University-Industry Joint Project on Quantum Nanostructures.

1. R. Heitz, et al., Appl. Phys. Lett. 68, 361 (1996), Phys. Rev. B 56, 10435 (1997).

\section{QTuC2}

8:15 am

Room-temperature dephasing in InAs/ GaAs quantum dots

P. Borri, W. Langbein, J.M. Hvam, M.-H. Mao, ${ }^{*}$ F. Heinrichsdorff,

D. Bimberg, ${ }^{*}$ Mikroelektronik Centret, The

Technical University of Denmark, Building 345 east, DK-2800 Lyngby, Denmark; E-mail: paola@mic.dtu.dk

Semiconductor quantum dots (QDs) are receiving increasing attention for fundamental studies on zero-dimensional confinement and for device applications. Quantum-dot lasers are expected to show superior performances, like high material gain, low and temperature-independent threshold current and chirp-free operation, ${ }^{1}$ due to the delta-like density of states (DOS)

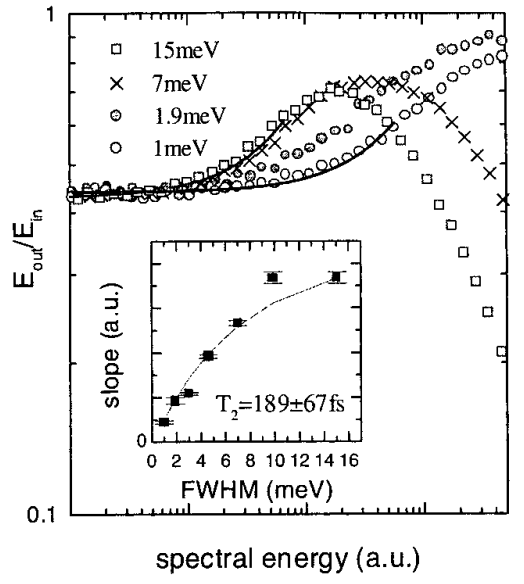

QTuC2 Fig. 1. QD transmission (in logarithmic scale) as a function of the input spectral energy for different spectral widths. The wavelength of the injected pulses was centered at $1.08 \mu \mathrm{m}$, corresponding to the ground state transition of the dots. Solid lines are fits to the data. In the inset, the slopes of the initial absorption bleaching and a fit according to our model are shown.

In this work we have measured the dephasing time at room temperature of InAs QDs embedded in a waveguide to estimate the lower limit for the energy-broadening of the DOS given by the homogeneous linewidth. The sample consists of 3 stacked layers of InAs/ InGaAs/GaAs quantum dots in the center of $120 \mathrm{~nm}$ GaAs embedded between two $\mathrm{Al}_{0.7} \mathrm{Ga}_{0.3}$ As cladding layers, ${ }^{2}$ in a ridge structure $8 \mu \mathrm{m}$ wide and $400 \mu \mathrm{m}$ long, with tilted facets. In Fig. 1 the responses of the device under injection of Fourier-limited optical pulses of different spectral widths are shown. The energy of the pulse at the output of the waveguide is measured by a $\mathrm{Ge}$-detector with lock-in technique. A bleaching of the absorption with increasing input intensity is clearly observed. Additionally, short pulses experience increasing absorption with input intensity due to two-photon absorption.

For a constant input spectral energy $E_{\omega}$ (energy per unit frequency) different bleaching occurs for different pulse-widths. This is simulated (solid lines in figure) by modeling the spectral hole-burning of the absorption coefficient induced by a pulse with a spectral width larger (open square) or smaller (open circle) than the homogeneous broadening of the dots. The bleaching shows a linear decrease of the absorption versus $\mathrm{E}_{\omega}$ with a slope that depends on pulse-width and dephasing time $T_{2}$. In the inset, the slopes obtained from the initial bleaching are shown, with a fit according to our model, giving $T_{2}=189 \pm 67 \mathrm{fs}$.

Another technique for measuring dephasing is four-wave mixing (FWM). ${ }^{3}$ FWM in thin films with spatial selection of the non-linear signal has been widely reported. However, FWM on $\operatorname{In}(\mathrm{Ga})$ As QDs has not yet been reported due to the weak signal from the small interaction volume and the large inhomogeneous broadening. We have used the heterodyne technique discussed in Ref. 4 to perform FWM in the waveguide geometry, with the advantage of using the entire length of the
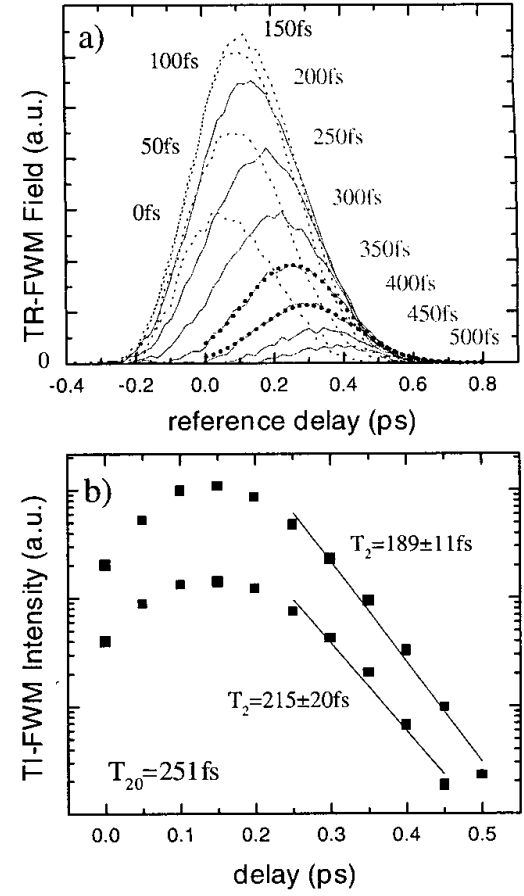

QTuC2 Fig. 2. (a) Time-resolved FWM electric field for different delay times of the two exciting beams. The photon-echo nature of the FWM is evident from the shift of the signal at long delay. Bold dotted lines are fits of the data. (b) Timeintegrated FWM intensity (electric field square) in the case of (a) (upper curve) and for half the excitation intensity (lower curve). Solid lines are fits of the data. The deduced dephasing times are indicated; $T_{20}$ is the extrapolated zero-density value.

device as interaction length. Figure 2(a) shows the amplitude of the time-resolved FWM electric field, by scanning over the delay of the reference beam, at different delay times $\tau$ of the exciting beams. Excitation and reference pulses had $200 \mathrm{fs}$ intensity-autocorrelation width. The photon-echo nature of the FWM is seen by the time-shift of the signal. A fit of the signal for $\tau=350,400 \mathrm{fs}$ is performed by using the formula ${ }^{3}: \mathrm{E}(\mathrm{t})=\mathrm{E}_{0} \mathrm{e}^{-\mathrm{t} / \mathrm{T}_{2}} \mathrm{e}^{-\sigma^{2}(\mathrm{t}-\tau)^{2} / 4}$ where $\sigma$ is 1.4 times the standard deviation of a Gaussian distribution. $\mathrm{T}_{2}=183 \pm 5$ fs is obtained. The integrated area of the FWM intensity is shown in Fig. 2(b) for the field reported in Fig. 2(a) and for half the excitation intensity. A fit of the decay is shown ${ }^{3}$ and the corresponding values of $T_{2}$ are indicated, together with the zero-density extrapolated value. These values are in good agreement with the ones reported in Fig. 1 within error bars.

${ }^{*}$ Insitut für Festkörperphysik, Technische Universität Berlin, Hardenbergstr. 36, 10623, Berlin, Germany

1. D. Bimberg et al., IEEE J. Selected Topics in Quantum Electronics 3, 1 (1997).

2. F. Heinrichsdorff et al., Appl. Phys. Lett. 71, 22 (1997).

3. J. Shah in Ultrafast Spectroscopy of Semiconductors and Semiconductor Nanostructures (Springer, Berlin 1996) Chap. 2.

4. M. Hoffmann et al., Applied Physics Letters 68, 3236 (1996). 